\title{
CLINIMEX Aerobic Fitness Questionnaire: Proposal and Validation
}

\author{
Claudio Gil S. Araújo, ${ }^{(\mathbb{C}}$ Claudia Lucia Castro, ${ }^{(\mathbb{1}}$ João Felipe Franca, ${ }^{(\mathbb{0}}$ Christina Grüne de Souza e Silva \\ Clínica de Medicina do Exercício - CLINIMEX, Rio de Janeiro, RJ - Brazil
}

\section{Abstract}

Background: Cardiorespiratory (aerobic) fitness is strongly and directly related to major health outcomes, including all-cause mortality. Maximum oxygen uptake $\left(\mathrm{VO}_{2} \mathrm{max}\right)$, directly measured by maximal cardiopulmonary exercise test (CPET), represents the subject's aerobic fitness. However, as CPET is not always available, aerobic fitness estimation tools are necessary.

Objectives: a) to propose the CLINIMEX Aerobic Fitness Questionnaire (C-AFQ); b) to validate C-AFQ against measured $\mathrm{VO}_{2}$ max; and c) to analyze the influence of some potentially relevant variables on the error of estimate.

Methods: We prospectively studied 1,000 healthy and unhealthy subjects (68.6\% men) aged from 14 to 96 years that underwent a CPET. The two-step C-AFQ describes physical activities with corresponding values in metabolic equivalents (METs) - ranging from 0.9 to 21 METs.

Results: Application of C-AFQ took less than two minutes. Linear regression analysis indicated a very strong association between estimated (C-AFQ) and measured (CPET) maximal METs - r2 = 0.83 (Sy.x = 1.63; p < .001) with median difference of only 0.2 METs between both values and interquartile range (percentiles 25 and 75) of 2 METs. The difference between estimated and measured METs was not influenced by age, sex, body mass index, clinical condition, ß-blocker use or sitting-rising test scores.

Conclusion: C-AFQ is a simple and valid tool for estimating aerobic fitness when CPET is unavailable and it is also useful in planning individual ramp protocols. However, individual error of estimate is quite high, so C-AFQ should not be considered a perfect substitute for CPET's measured $\mathrm{VO}_{2}$ max. (Int J Cardiovasc Sci. 2019;32(4):331-342)

Keywords: Exercise; Breathing Exercises; Exercise Test; Exercise Therapy; Health Impact; Validation Studies; Surveys and Questionnaires.

\section{Introduction}

Several long-term cohort studies have clearly shown a strong and direct association between cardiorespiratory (aerobic) fitness and a better and longer survival in adult men and women from different countries. ${ }^{1-3}$ Quantified in $\mathrm{mL} \mathrm{O}_{2} \cdot \mathrm{kg}^{-1} \cdot \mathrm{min}^{-1}$ or simply as metabolic equivalents or METs $\left(1 \mathrm{MET}=3.5 \mathrm{~mL} \mathrm{O}_{2} \cdot \mathrm{kg}^{-1} \cdot \mathrm{min}^{-1}\right)$, aerobic fitness is also associated with lower chances of developing major clinically relevant diseases, such as coronary artery disease, arterial hypertension and several types of cancer. ${ }^{4-6}$ Additionally, functional capacity, which is strongly related to aerobic fitness, has also been recently recognized as a clinical vital sign. ${ }^{7}$ 
Perhaps, in a clinical context, there is no other variable that outweighs aerobic fitness in terms of relevance to major adverse outcomes such as cardiovascular, cancer and all-cause mortality ${ }^{8}$ and each 1 MET increase in aerobic fitness is associated with long-term risk reduction of 10 to $15 \%$ chance of dying. ${ }^{9,10}$ Even more interesting, a recent analysis of important cohorts in United States and Finland has shown that middle-aged or older men that improve their aerobic fitness over time tend to substantially decrease their mortality rate. ${ }^{11-13}$

The gold standard for aerobic fitness determination is the measurement of maximum oxygen uptake $\left(\mathrm{VO}_{2} \max \right)$ during maximal cardiopulmonary exercise test (CPET), ${ }^{7,14,15}$ by progressively increasing exercise intensity in an ergometer, most often a treadmill or a leg cycle ergometer, until volitional exhaustion, while collecting and analyzing expired gases. However, despite the existence of several institutional guidelines, ${ }^{14,16}$ for a number of reasons, the use of CPET for quantifying aerobic fitness remains quite limited around the world and in Brazil. In this context, non-exercise alternatives to estimate aerobic fitness could be worth exploring. Moreover, even when CPET is available, estimating aerobic fitness would help to plan a more precise ramp protocol, that is, initial and incremental rate per minute in watts or speed/slope for a maximal CPET that will last around 10 minutes. ${ }^{17}$

In a study with 63 subjects, ${ }^{18}$ it was found that using measured $\mathrm{VO}_{2} \max$ as the gold standard, well-educated adults were reasonably well capable of classifying themselves as having much lower, lower, similar, higher or much higher aerobic fitness of what should be expected for their sex- and age-matched peers. In a classical study, researchers ${ }^{19}$ have proposed the Veterans Specific Activity Questionnaire (VSAQ) to estimate aerobic fitness, obtaining good association $-r=0.79$ between VSAQ and measured $\mathrm{VO}_{2}$ max. The VSAQ has been largely used ${ }^{20-22}$ and it has been transculturally adapted to Brazil ${ }^{23,24}$ with reasonable results. However, despite several merits, the VSAQ also has some important limitations: 1) it was primarily validated in a sample of middle-aged and old men; 2 ) the relatively long-time needed for the subject to read all the 21 lines in order to classify him(her)self; 3) the upper limited score - 13 METs - that excludes many healthy exercisers and athletes; and 4) the unique one-MET interval across all scales, potentially losing discrimination for those placed in the lower range of aerobic fitness.
Therefore, it seems an interesting proposal to develop a Brazilian questionnaire for estimating aerobic fitness that would be culturally adjusted to its population and that would circumvent the main limitations of VSAQ. The performance of CPET in a well-controlled setting, in men and women presenting a large age range and extremes of aerobic fitness, offered an outstanding and unique research opportunity to prospectively assess the validity of a new questionnaire, the CLINIMEX Aerobic Fitness Questionnaire (C-AFQ).

The objectives of this study were: a) to propose the $\mathrm{C}-\mathrm{AFQ}$; b) to validate C-AFQ against the gold standard measured $\mathrm{VO}_{2} \max ; \mathrm{c}$ ) to compare the physician's statistical error of estimating aerobic fitness by C-AFQ; and d) to analyze the influence of age, sex, clinical conditions, regular use of $\beta$-blockers and two non-aerobic fitness test scores on the error of estimate of aerobic fitness by $\mathrm{C}-\mathrm{AFQ}$.

\section{Methods}

\section{Study sample}

Prospective data collection started in January $5^{\text {th }} 2016$ and was planned to continue until data from a total of 1,000 subjects was obtained. As previously defined in the research design, subjects younger than 14 or athletes ${ }^{25}$ or those not completing a true maximal cardiopulmonary exercise test (CPET) were not included in the study. ${ }^{26,27} \mathrm{In}$ addition, those with any missing or incomplete relevant data were also excluded. The final sample of 1,000 subjects was completed in May $7^{\text {th }} 2019$. The vast majority of subjects (98\%) were white and pertaining to a high socioeconomic class. All subjects voluntarily went to our Clinic for the evaluation protocol. Before the evaluation, all subjects read and signed a specific informed consent form previously approved by the institutional committee on ethics in research.

The final sample included 686 men $(68.6 \%)$ aged 14 to 96 years (mean \pm standard deviation: $55.2 \pm 16.4$ years). From the total of 1,000 subjects studied, $72.5 \%$ were evaluated for the first time in our Clinic, while the remaining $27.5 \%$ had been evaluated between two and 20 times. Regarding the subjects' clinical conditions, $22 \%$ were considered healthy (no cardiorespiratory or major diseases reported), $23.3 \%$ had a diagnosis of coronary artery disease, including $12.2 \%$ with previous myocardial infarction, $15.2 \%$ that had been submitted to coronary angioplasty and 5.9\% to coronary artery 
bypass grafting. A total of $34.7 \%$ were being treated for arterial hypertension, $40.2 \%$ were classified as having dyslipidemia, and diabetes mellitus was diagnosed in $13.4 \%$ of the subjects studied. Regular use of ß-blockers was reported by $25.6 \%$ of the subjects.

\section{Maximal cardiopulmonary exercise test (CPET)}

Only four experienced and specialized physicians directly supervised all maximal CPETs performed in a proper temperature-humidity controlled room equipped for providing medical emergency support if needed. Digital electrocardiogram was continuously monitored and recorded before, during and for at least five minutes after the end of the CPET (Micromed Elite ErgoPC, Brazil).27,28 The ergometer was chosen according to the subject's testing objective, whether for clinical diagnosis, exercise prescription or sport training advice. Maximal CPET was most often performed in leg cycling ergometer (85.2\%) (Inbramed CG-04, Brazil) than in treadmill (14.8\%) (Inbramed ATL Master, Brazil) using individual ramp protocols. For treadmill tests, a constant $0 \%$ slope was set and after one-minute walk at $5.5 \mathrm{~km} / \mathrm{h}$, the speed was quickly increased to $8.0 \mathrm{~km} / \mathrm{h}$ and thereafter progressively increased until volitional exhaustion under strong verbal encouragement. For all CPETs, individualized rates of exercise intensity - speed or watts - increment was used. A combination of several physiological and perceptual criteria was utilized for characterizing CPET as maximal in both ergometers. ${ }^{27,28}$ No major relevant clinical abnormalities occurred during all 1,000 CPETs performed.

During the CPET, subjects used a nose clip and breathed through a Prevent mouthpiece in order to collect expired gas. Calculation of air flow and $\mathrm{O}_{2}$ and $\mathrm{CO}_{2}$ expired fraction analysis were carried out in a VO2000 metabolic analyzer (Medical Graphics, United States) regularly calibrated using known syringe volumes and two different gas concentrations.

For comparison purposes, $\mathrm{VO}_{2}$ max related to body weight in cycling CPET was predicted according to age and sex by using the following equations proposed by Jones et al. ${ }^{29}$ : men $=60-0.55 \mathrm{x}$ age (years) and for women $=48-0.37 \times$ age (years). For treadmill CPET, $11 \%$ was added to this predicted value. All data related to $\mathrm{VO}_{2} \mathrm{max}$ - predicted, measured and estimated by $\mathrm{C}-\mathrm{AFQ}$ - were reported as METs, where $1 \mathrm{MET}=3.5$ $\mathrm{mL} \mathrm{O}_{2} \cdot \mathrm{kg}^{-1} \cdot \mathrm{min}^{-1}$.

\section{CLINIMEX aerobic fitness questionnaire (C-AFQ)}

C-AFQ data were obtained during consultation with the supervising physician either by objective questioning or by showing the list of activities and corresponding METs. C-AFQ was applied in a two-step sequence following standard instructions (please see footnote of Table 1 for more details). (see Portuguese C-AFQ version in supplemental materials). Almost all the corresponding MET values for each of the listed activities were obtained from the literature,,$^{30}$ with few of them estimated from other sources or by the authors' scientific knowledge and clinical experience.

This two-step approach allowed refining the estimate of maximal exercise capacity and, consequently, maximal aerobic power in METs. By applying C-AFQ, it was possible to estimate aerobic fitness in one or two minutes, from $<1$ to $>20$ METs, with 0.5 intervals from 2 to 5 METs and thereafter, from one in one MET increments up to 20 or more METs.

\section{Other study measurements}

Our evaluation protocol was quite comprehensive and involved several other variables tests. In order to identify potential influential variables for this particular study, age, sex, height, weight, waist girth, ${ }^{31}$ sitting-rising tes ${ }^{32,33}$ and maximal muscle power related to body weight in the upper row movement ${ }^{34}$ were also tabulated and analyzed.

\section{Statistical analysis}

Descriptive statistics for continuous variables were presented as mean and standard deviations or as median and interquartile range, as well as several other potentially useful percentiles for data distribution and frequency distribution for nominal variables. Inferential statistics analyses were carried out by t-tests or ANOVA and Tukey's post-hoc comparisons, depending on the number of groups compared. Pearson product-moment correlation coefficients were calculated for assessing association and best-fit linear regression was used for data modeling. Statistical significance was set at $5 \%$ of probability and the Prism version 8.1.1 software package (GraphPad, United States) was used for statistical calculations and preparation of figures.

\section{Results}

Sex- and age-predicted $\mathrm{VO}_{2}$ max and measured (CPET) $\mathrm{VO}_{2}$ max were similar, being, respectively, 
Table 1 - Application instructions

1 The C-AFQ (CLINIMEX Aerobic Fitness Questionnaire) should be applied in two steps, to make it faster and more efficient.

The objective of step 1 is to identify the range to which the individual belongs (0 to 10$)$, by asking: - Do you think you can do this?

2 If the answer is positive, move to the following sentence from the Zone immediately below, until a negative response is obtained. Otherwise, go back to the previous zone.

To speed up, the interviewer can already pre-select zones, as suggested below

if the individual is less than 40 years old (male) - Zone Line 5

if he is between 51 and 60 years old - Zone Line 4

if he is over 60 - Zone Line 3

Once the correct zone is identified, proceed to step 2

4 In step 2, the various sub-band options are presented and the individuals should identify which of the sentences he/ she believes it could do (it can be more than one or just one of the sentences).

The estimated aerobic fitness is characterized by the number of METs corresponding to the MET value that contains a sentence with any exercise or physical activity he/ she believes that it will be able to perform.

Table 2 - CLINIMEX - Aerobic Fitness Questionnaire

\section{AEROBIC FITNESS QUESTIONNAIRE [step 1]}

Instruction to fill:

Identify the number corresponding to the most intense exercise / physical activity you are likely to do with your current aerobic fitness (disregarding recent orthopedic problems or other relevant motion limitations)

\begin{tabular}{|c|c|c|}
\hline ZONE & Exercise or Physical Activity & METs \\
\hline 0 & Lying in bed & 0.9 \\
\hline 1 & Sitting: napping, reading, watching television or listening to the radio & 1 \\
\hline 2 & Standing: working, talking, ironing, cooking or attending religious acts/shows/plays & 2 \\
\hline 3 & Walking at least $1 \mathrm{~km}$ or 10 minutes (non-stop) & 3.5 \\
\hline 4 & Running, slowly, one block or 100 meters & 6 \\
\hline 5 & Running, slowly, at least $1 \mathrm{~km}$ or 10 minutes (no walking or stopping) & 9 \\
\hline 6 & Running continuously, for up to 40 minutes & 12 \\
\hline 7 & Completing a half-marathon in about 2 hours and a marathon in about $4 \frac{1}{2}$ hours & 15 \\
\hline 8 & Completing a half-marathon in $1 \mathrm{~h} 30 \mathrm{~min}-1 \mathrm{~h} 40 \mathrm{~min}$ or a marathon in $3 \mathrm{~h} 15 \mathrm{~min}-3 \mathrm{~h} 40 \mathrm{~min}$ & 18 \\
\hline 9 & Completing a half-marathon in $1 \mathrm{~h} 20 \mathrm{~min}-1 \mathrm{~h} 30 \mathrm{~min}$ or a marathon in $3 \mathrm{~h}-3 \mathrm{~h} 15 \mathrm{~min}$ & 20 \\
\hline 10 & Running a half-marathon in less than $1 \mathrm{~h} 20 \mathrm{~min}$ or a marathon for less than 3 hours & $>20$ \\
\hline
\end{tabular}

$8.41 \pm 0.08$ METs [mean \pm standard error of the mean] and $8.56 \pm 0.12$ METs $(\mathrm{p}=0.07)$. However, for a given individual, measured $\mathrm{VO}_{2}$ max could range from $28.8 \%$ to $236 \%$ of sex- and age-predicted $\mathrm{VO}_{2} \max$, corresponding to differences ranging from -9.6 to 8.3 METs. The correlation coefficient between the sex- and age-predicted $\mathrm{VO}_{2}$ max and measured $\mathrm{VO}_{2}$ max was 0.69 $(\mathrm{p}<0.001)$.
The minimum and maximum values obtained from the 1,000 subjects for C-AFQ and measured $\mathrm{VO}_{2}$ max were, respectively, 2 and 21 METs and 1.9 and 20.7 METs, with interquartile ranges [percentile 25-percentile 75] being 6.0-12.0 METs for estimated and 5.6-11.0 METs for measured aerobic fitness. When comparing the mean values, a small but statistically significant difference was found between estimated aerobic fitness $-8.92 \pm 0.12$ 
Table 3 - CLINIMEX - Aerobic Fitness Questionnaire

\section{AEROBIC FITNESS QUESTIONNAIRE [step 2]}

Instruction to fill:

Check the maximum or most intense exercise/physical activity that best represents what you would be able to perform with your current aerobic fitness (disregarding recent orthopedic problems or other relevant motion limitations)

\begin{tabular}{llrr}
\hline METs & Exercise or Physical Activity & ZONE & METS \\
\hline 0,9 & Lying in bed & 0 & $<1$ \\
\hline
\end{tabular}

1 Sitting: napping, reading, watching television or listening to the radio

$1 \quad$ Meditating

Sitting: dressing or typing / playing on the cell phone or computer

1,5 Sitting: eating or talking or attending Religious Services or watching games or sports competitions on site Sitting: playing cards or chess or bathing yourself

Standing: working, talking, ironing, cooking or religious act/show/play

2 Caring for the elderly / babies or sewing or bathing alone while standing

Participate in Pilates, Hatha Yoga or water aerobics classes (very slow pace)

Maintaining sexual intercourse (more passive and less intense participation)

Walking at a slow pace $-3.6 \mathrm{~km} / \mathrm{h}(60 \mathrm{~m} / \mathrm{min})$

Playing musical instruments (sitting) or singing while standing

2,5 Cleaning the house with non-motorized equipment or devices

Driving cars with manual transmission in local transit

Attending stretching or bodybuilding classes, alternating the exercises with rest breaks

Walking at a normal pace $-4.8 \mathrm{~km} / \mathrm{h}(80 \mathrm{~m} / \mathrm{min})$

3 Attending Pilates, Hatha Yoga or aqua fitness classes (at a moderate pace)

Singing out loud or playing musical instruments while standing

Maintaining sexual intercourse (more active and very intense participation)

Walking at least $1 \mathrm{~km}$ or 10 minutes (non-stop)

3,5 Car washing or heavy domestic housecleaning

Working with equipment or instruments weighing between 1 and $5 \mathrm{~kg}$

Brisk walking at $6 \mathrm{~km} / \mathrm{h}(100 \mathrm{~m} / \mathrm{min})$

$4 \quad$ Playing (intense activities) with children or pets

Attending Pilates classes, yoga, dance or aqua fitness classes (at a fast pace)

Engaging in slow dancing activities

Fast walking at a very fast pace at $6.5-7 \mathrm{~km} / \mathrm{h}(108-118 \mathrm{~m} / \mathrm{min}$ or $1 \mathrm{~km}$ in 8 to 9 minutes)

$4,5 \quad$ Walking on a slope (up to $3 \%$ ) *

Playing ball or racquet sports on courts or on the sand for recreational purposes (low intensity)

Walking on a slope (up to $5 \%$ ) *

5 Brisk walking at $6 \mathrm{~km} / \mathrm{h}(100 \mathrm{~m} / \mathrm{min})$ carrying between 5 and $10 \mathrm{~kg}$ (child, shopping, equipment and similar)

Engaging in fast dancing activities (ballroom dancing, rock, funk or similar)

\begin{tabular}{ll}
\hline $6 \quad \begin{array}{l}\text { Running slowly one block or } 100 \text { meters } \\
\text { Attending high-intensity workout classes } \\
\text { Pedaling recreationally or pedaling to work / school for up to half an hour }\end{array}$ \\
\hline $7 \quad \begin{array}{l}\text { Running at a slow pace for } 1 \text { to } 3 \text { minutes } \\
\text { Walking on moderately inclined trails ( } 5 \text { to } 10 \% \text { on average) * } \\
\text { Engaging in very fast dancing styles (salsa, samba, merengue, tango and alike) }\end{array}$ \\
\hline $\begin{array}{l}\text { Running at a slow pace for } 4 \text { to } 6 \text { minutes } \\
\text { Playing recreationally or attending a (single) tennis lesson or ball sports for over an hour } \\
\text { Pedaling, outdoors or road, between } 16 \text { to } 20 \mathrm{~km} / \mathrm{h} \text { or to go to work/ school for up to one hour }\end{array}$ \\
\hline
\end{tabular}




\section{Continuation Table 3}

\begin{tabular}{|c|c|c|c|}
\hline 9 & $\begin{array}{l}\text { Running slowly at least } 1 \mathrm{~km} \text { or } 10 \text { minutes (no walking or stopping) } \\
\text { Attending spinning or step aerobics classes (low or moderate intensity) } \\
\text { Engaging in martial arts for at least one hour with short breaks only }\end{array}$ & \multirow{3}{*}{5} & \multirow{3}{*}{9 to 11.9} \\
\hline 10 & $\begin{array}{l}\text { Running continuously for } 10 \text { to } 15 \text { minutes } \\
\text { Running for at least one minute at } 10 \mathrm{~km} / \mathrm{h}(167 \mathrm{~m} / \mathrm{min}) \text { outdoors or } 10.5 \mathrm{~km} / \mathrm{h} \text { on the treadmill } \\
\text { Pedaling outdoors or on the road at } 20 \text { and } 25 \mathrm{~km} / \mathrm{h} \text { for up to half an hour }\end{array}$ & & \\
\hline 11 & $\begin{array}{l}\text { Running at least one minute at } 11 \mathrm{~km} / \mathrm{h}(184 \mathrm{~m} / \mathrm{min}) \text { outdoors or } 11.6 \mathrm{~km} / \mathrm{h} \text { on the treadmill } \\
\text { Attending fast-pedaling spinning or running classes }\end{array}$ & & \\
\hline 12 & $\begin{array}{l}\text { Running continuously for up to } 40 \text { minutes } \\
\text { Running for at least one minute at } 12 \mathrm{~km} / \mathrm{h}(200 \mathrm{~m} / \mathrm{min}) \text { outdoors or } 12.7 \mathrm{~km} / \mathrm{h} \text { on the treadmill } \\
\text { Playing ball sports at very intense pace and for at least } 30 \text { minutes without breaks }\end{array}$ & \multirow{3}{*}{6} & \multirow{3}{*}{12 to 14.9} \\
\hline 13 & $\begin{array}{l}\text { Running for at least one minute at } 13 \mathrm{~km} / \mathrm{h}(200 \mathrm{~m} / \mathrm{min}) \text { outdoors or } 13.8 \mathrm{~km} / \mathrm{h} \text { on the treadmill } \\
\text { Running } 10 \mathrm{~km} \text { in about } 1 \text { hour } \\
\text { Pedaling outdoors or on the road at } 20 \text { and } 25 \mathrm{~km} / \mathrm{h} \text { for more than one hour }\end{array}$ & & \\
\hline 14 & $\begin{array}{l}\text { Running for at least one minute at } 14 \mathrm{~km} / \mathrm{h}(233 \mathrm{~m} / \mathrm{min}) \text { outdoors or } 14.9 \mathrm{~km} / \mathrm{h} \text { on the treadmill } \\
\text { Running } 10 \mathrm{~km} \text { in } 53 \text { to } 57 \text { minutes or completing a half-marathon in about } 2 \mathrm{~h} 15 \mathrm{~min}\end{array}$ & & \\
\hline 15 & $\begin{array}{l}\text { Running a half-marathon in about } 2 \text { hours or a marathon in about } 4 \frac{1}{1 / 2} \text { hours } \\
\text { Running for at least one minute at } 15 \mathrm{~km} / \mathrm{h}(250 \mathrm{~m} / \mathrm{min}) \text { outdoors or } 16 \mathrm{~km} / \mathrm{h} \text { on the treadmill }\end{array}$ & \multirow{3}{*}{7} & \multirow{3}{*}{15 to 17.9} \\
\hline 16 & $\begin{array}{l}\text { Running for at least one minute at } 16 \mathrm{~km} / \mathrm{h}(266 \mathrm{~m} / \mathrm{min}) \text { outdoors or } 17.2 \mathrm{~km} / \mathrm{h} \text { on the treadmill } \\
\text { Running a half-marathon in about } 1 \mathrm{~h} 50 \mathrm{~min} \text { to } 1 \mathrm{~h} 55 \mathrm{~min} \text { or a marathon in } 4 \mathrm{~h} \text { to } 4 \mathrm{~h} 15 \mathrm{~min}\end{array}$ & & \\
\hline 17 & $\begin{array}{l}\text { Running for at least one minute at } 17 \mathrm{~km} / \mathrm{h}(283 \mathrm{~m} / \mathrm{min}) \text { outdoors or } 18.4 \mathrm{~km} / \mathrm{h} \text { on the treadmill } \\
\text { Running a half-marathon in about } 1 \mathrm{~h} 40 \mathrm{~min} \text { or a marathon in } 3 \mathrm{~h} 40 \mathrm{~min} \text { to } 4 \mathrm{~h}\end{array}$ & & \\
\hline 18 & $\begin{array}{l}\text { Running a half-marathon in } 1 \mathrm{~h} 30 \mathrm{~min} \text { to } 1 \mathrm{~h} 40 \mathrm{~min} \text { or a marathon in } 3 \mathrm{~h} 15 \mathrm{~min} \text { to } 3 \mathrm{~h} 40 \mathrm{~min} \\
\text { Running for at least one minute at } 18 \mathrm{~km} / \mathrm{h}(300 \mathrm{~m} / \mathrm{min}) \text { outdoors or } 19.6 \mathrm{~km} / \mathrm{h} \text { on the treadmill }\end{array}$ & 8 & 18 to 19.9 \\
\hline 20 & $\begin{array}{l}\text { Running a half-marathon in } 1 \mathrm{~h} 20 \mathrm{~min} \text { to } 1 \mathrm{~h} 30 \mathrm{~min} \text { or a marathon in } 3 \mathrm{~h} \text { to } 3 \mathrm{~h} 15 \mathrm{~min} \\
\text { Running for at least one minute at } 20 \mathrm{~km} / \mathrm{h}(333 \mathrm{~m} / \mathrm{min}) \text { outdoors or } 22 \mathrm{~km} / \mathrm{h} \text { on the treadmill }\end{array}$ & 9 & 20 \\
\hline$>20$ & Running a half-marathon in less than $1 \mathrm{~h} 20 \mathrm{~min}$ or a marathon in less than 3 hours & 10 & $>20$ \\
\hline
\end{tabular}

METs - and measured aerobic fitness - $8.56 \pm 0.12$ METs - $(\mathrm{p}<0.001)$, with standard error of the mean differences of only 0.05 METs. The median of the difference between estimated and measured aerobic fitness was only 0.2 METs with interquartile range from - 0.7 to 1.3 METs (see violin plot in Figure 1). Table 4 describes separately men and women's data for some of these results.

A very direct and strong association was found between estimated (C-AFQ) and measured (CPET) $\mathrm{VO}_{2}$ max values $-\mathrm{r}=0.91(\mathrm{p}<0.001)$. Linear regression analysis found a coefficient of determination or $\mathrm{r}^{2}$ value of $0.833(\mathrm{p}<0.001)$ and a standard error of estimate of 1.54 METs. The scatterplot of 1,000 subject's data, identity line and 2-MET individual difference lines are presented in Figure 2.
Considering that four different physicians have applied the C-AFQ for the 1,000 subjects, we analyzed estimated and measured aerobic fitness values and their respective differences for each one of them in order to search for any applicant C-AFQ bias. Median and interquartile measured METs were: physicians 1-8.7 [6.1-11.1], physician 2-6.8 [5.0-9.3], physician 3-7.9 [5.7-11.8] and physician 4-7.7 [4.9-13.7] $(\mathrm{p}<0.001)$. Despite these quite distinct levels of aerobic fitness in the subjects evaluated by the four physicians, C-AFQ values were quite similar among them $(p=0.055)$ and multiple comparison analysis identified that the only the physician's pair 1-2 significantly differed (Figures 3 and 4) - exactly the two physicians that were in the extremes of median values for measured aerobic fitness. 


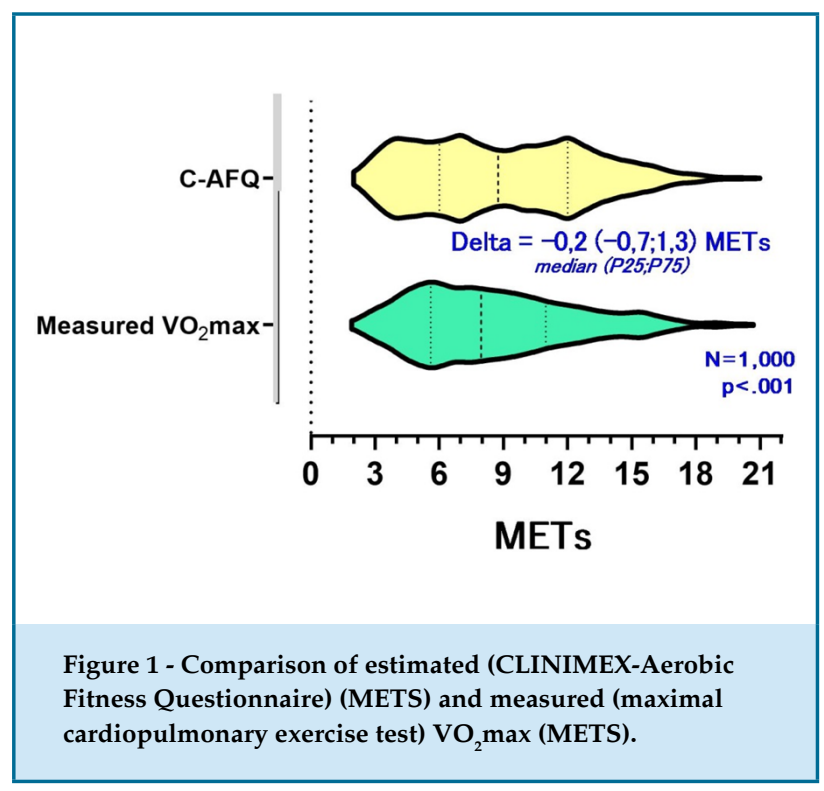

Table 4 - Descriptive statistics for men and women for main variables*

\begin{tabular}{|c|c|c|}
\hline & Men & Women \\
\hline $\mathrm{N}$ & 687 & 313 \\
\hline Age (years) & $\begin{array}{c}56.2 \pm 16.5 \\
{[34-78]}\end{array}$ & $\begin{array}{c}53.1 \pm 16.2 \\
{[31-75]}\end{array}$ \\
\hline Height (cm) & $\begin{array}{c}175.1 \pm 7.5 \\
{[165.1-184.5]}\end{array}$ & $\begin{array}{c}161.9 \pm 7.1 \\
{[153.4-171.9]}\end{array}$ \\
\hline Weight (kg) & $\begin{array}{c}84.5 \pm 14.9 \\
{[68.8-103.4]}\end{array}$ & $\begin{array}{c}67.0 \pm 12.7 \\
{[52.8-84.8]}\end{array}$ \\
\hline Body mass index $\left(\mathrm{kg} / \mathrm{m}^{2}\right)$ & $\begin{array}{c}27.5 \pm 4.2 \\
{[23.0-32.5]}\end{array}$ & $\begin{array}{c}25.6 \pm 4.9 \\
{[20.4-31.9]}\end{array}$ \\
\hline Waist** $\left.^{*} \mathrm{~cm}\right)$ & $\begin{array}{c}97.5 \pm 12.6 \\
{[83.0-113.7]}\end{array}$ & $\begin{array}{c}85.5 \pm 12.9 \\
{[70.8-103.5]}\end{array}$ \\
\hline Measured $\mathrm{VO}_{2}(\mathrm{METs})$ & $\begin{array}{l}9.02 \pm 3.85 \\
{[4.5-14.9]}\end{array}$ & $\begin{array}{l}7.54 \pm 3.35 \\
{[3.5-12.3]}\end{array}$ \\
\hline C-AFQ (METs) & $\begin{array}{l}9.35 \pm 4.02 \\
{[4.0-15.0]}\end{array}$ & $\begin{array}{l}7.98 \pm 3.62 \\
{[3.5-13.0]}\end{array}$ \\
\hline Predicted $\mathrm{VO}_{2}$ (METs) & $\begin{array}{l}8.48 \pm 2.77 \\
{[4.9-12.2]}\end{array}$ & $\begin{array}{l}8.26 \pm 1.86 \\
{[5.8-11.0]}\end{array}$ \\
\hline $\begin{array}{l}\text { * data expressed as mean } \pm s \\
\text { percentile } 90] ; * * \text { measured }\end{array}$ & $\begin{array}{l}\text { d deviation ar } \\
\text { ilical level. }\end{array}$ & rcentile 10 - \\
\hline
\end{tabular}

In order to analyze the potential influence of several other variables in the magnitude of the main result, we used two different approaches. First, we compared results for the delta METs, i.e., the difference between

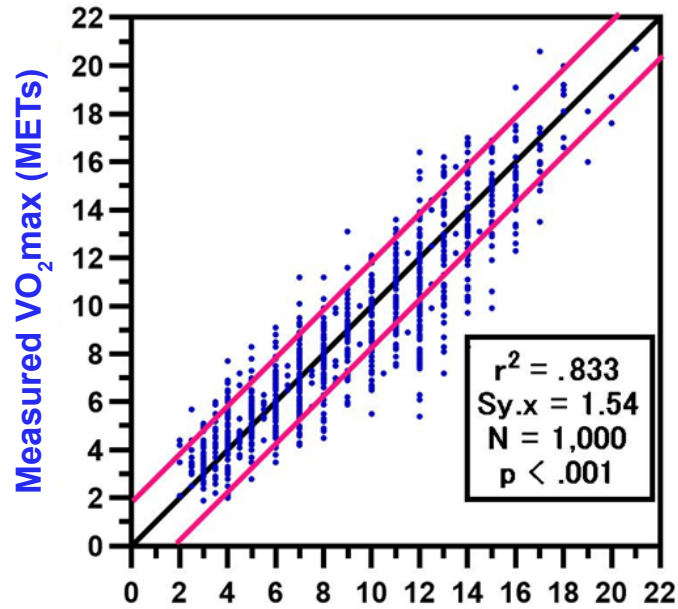

\section{CLINIMEX AFQ (METs)}

Figure 2 - Linear regression and scatterplot of measured $\mathrm{VO}_{2} \max$ (METs) and estimated $\mathrm{VO}_{2} \max$ (METS) by CLINIMEX Aerobic Fitness Questionnaire.

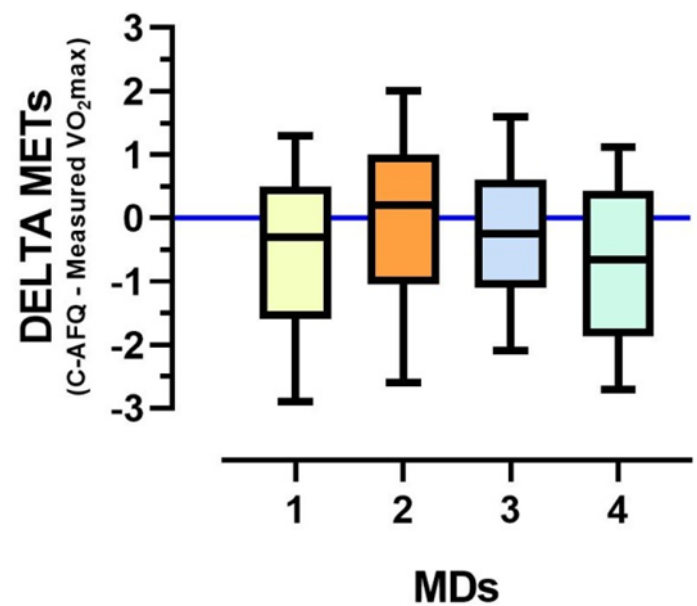

Figure 3 - Separate comparison of measured $\mathrm{VO}_{2} \max$ (METs) and estimated $\mathrm{VO}_{2} \max$ (METS) by CLINIMEX-Aerobic Fitness Questionnaire among the four physicians. Footnote: Boxes represent percentiles $25-75$ and whiskers limit percentiles 10 and 90; central boxes' line represent median value.

estimated (C-AFQ) and measured (CPET) $\mathrm{VO}_{2}$ max, dividing the total sample into two subgroups according to sex (men vs. women), clinical condition (healthy vs. unhealthy), regular use of ß-blockers (yes vs. no) 
95\% Confidence Intervals (Tukey)

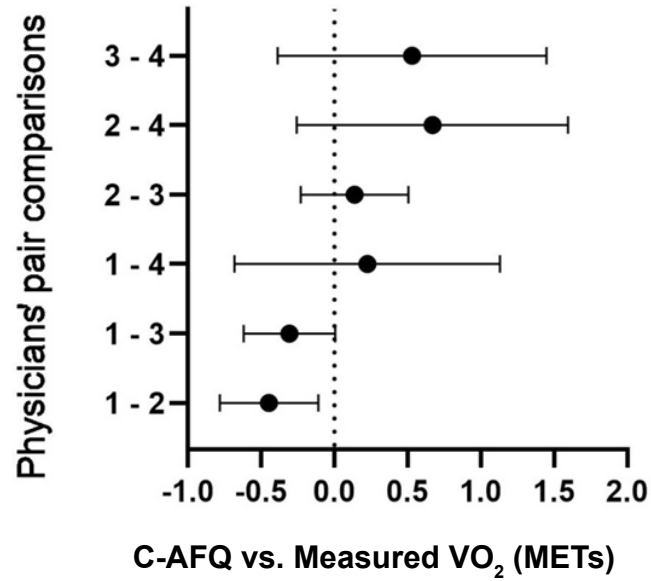

Figure 4 - Multiple comparisons for the delta METs among the four physicians. and ergometer (treadmill vs. cycling). Unpaired t-tests showed that none of these variables were found to be relevant in influencing the magnitude of delta METs, with p-values of 0.31 for sex, 0.52 for clinical condition, 0.21 for $ß$-blocker regular use and 0.05 for ergometer.

In the second approach, a matrix correlation was calculated. Figure 5 presents the major association results in the format of a heatmap, in which white boxes indicate non-significant correlation coefficients and stronger coloration reflects more association and higher $\mathrm{r} 2$ values; additionally, blue and red color indicated, respectively, positive and negative correlation coefficients. As expected, measured $\mathrm{VO}_{2} \max$ was inversely related to age $-r=-0.63(p>0.001)$. Similar or slightly higher correlation coefficients were obtained for the associations between the scores of the two non-aerobic fitness tests and the measured $\mathrm{VO}_{2}$ max.

Regarding delta METs, i.e., the difference between estimated (C-AFQ) and measured (CPET) $\mathrm{VO}_{2}$ max, we

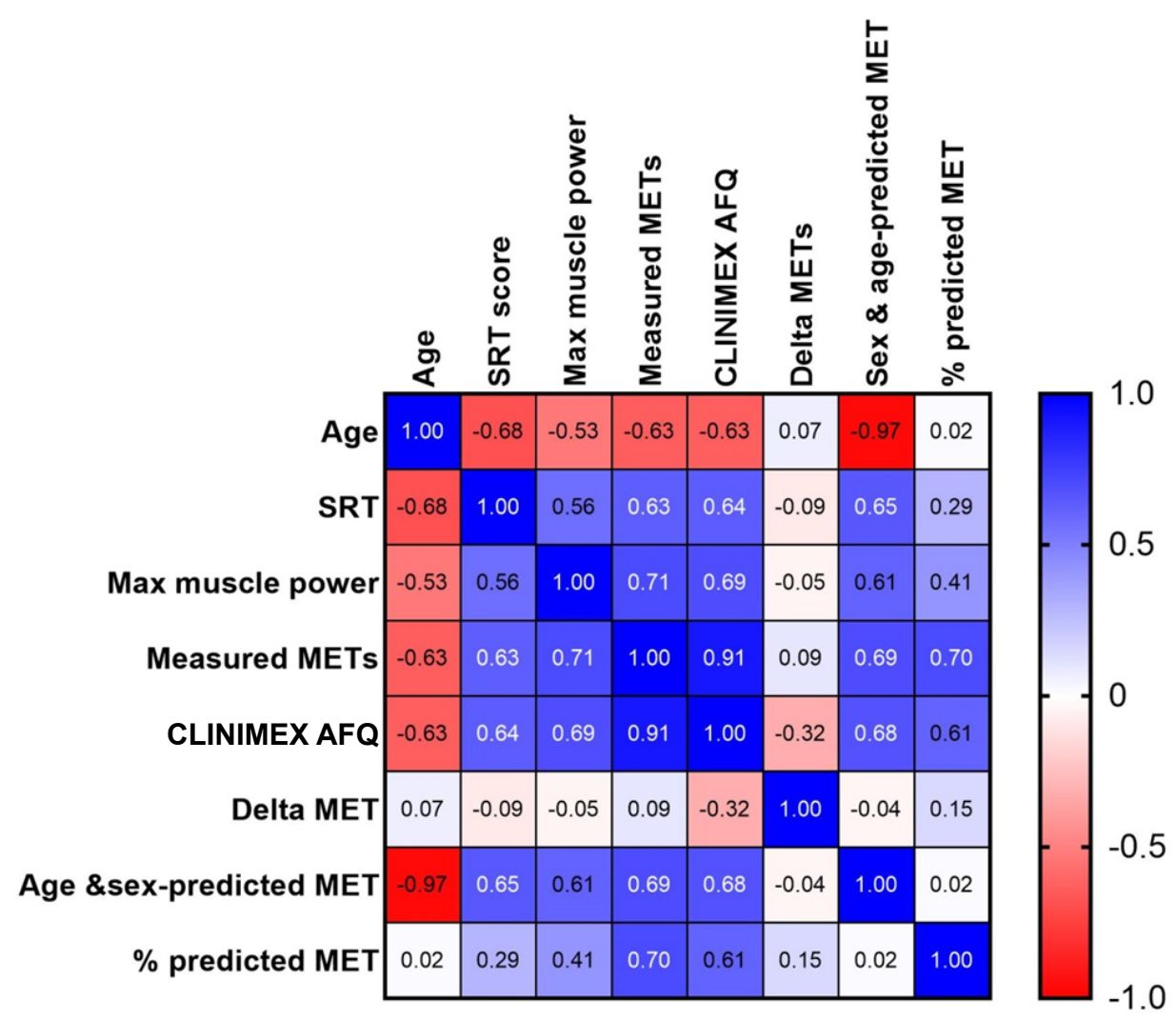

Figure 5 - Heatmap of the associations found for the main study variables. 
found non-significant associations ( $\mathrm{r}$ values $>-0.1$ and $<0.1 ; \mathrm{p}>0.05$ ) with age and the anthropometric variables studied - height, weight, waist and body mass index (these last two not represented in figure 2). The r-values between delta METs with other non-aerobic components of physical fitness, as assessed by SRT scores and maximal muscle power were, respectively, -0.09 and -0.04 , also suggesting no clinical relevance or implications.

The small but significant and positive associations found between delta METs and measured METs $r=0.09(p<0.001)-$ and measured METs expressed as $\%$ sex- and age-predicted METs $-\mathrm{r}=0.15(\mathrm{p}<0.001)$, but not with sex- and age-predicted MET $-\mathrm{r}=-0.04$ $(p=0.55)$, indicated that the delta METs slightly tends to increase for those subjects at an upper range of measured aerobic fitness. Median [percentiles 25-75] CPET duration was 11 [8-12] minutes with $75 \%$ of all 1,000 CPETs lasting between 8 and 15 minutes.

\section{Discussion}

This study proposed the C-AFQ, a new assessment tool for non-exercise estimation of aerobic fitness, and also validated it against the gold standard and criterion measurement of $\mathrm{VO}_{2}$ max obtained during a maximal CPET. In addition, it explored the potential influence of several other variables in the magnitude of the delta METs between estimated (C-AFQ) and measured (CPET) $\mathrm{VO}_{2}$ max.

The rationale for proposing the C-AFQ was primarily based on two major needs: a) to have a simple and valid tool for estimating $\mathrm{VO}_{2}$ max for the Brazilian population when CPET was not feasible or desirable to be carried out; and $b$ ) to obtain subsidies for a better and more precise planning of an individual CPET ramp protocol.

Regarding the main variable of the study, a small yet statistically significant difference was found between both means and medians of estimated and measured $\mathrm{VO}_{2}$ max, being, respectively, 0.38 and 0.2 METs. However, considering the minimal interval of $0.5 \mathrm{MET}$ in the C-AFQ scale, these small differences were likely to be clinically irrelevant and acceptable. It should also be recognized that in some subjects that were more used to running than to cycling exercises, the clinical option (i.e., need for a more precise measurement of exercise blood pressure) of cycling rather than treadmill CPET might have produced some overestimation of estimated $\mathrm{VO}_{2}$ max when the C-AFQ was applied, and therefore, contributed to the modest yet significant difference found between the means and medians. Additionally, it should also be mentioned that in some cases, the use of C-AFQ was limited either by not contemplating some specific regular exercises, such as primarily swimming and rowing, or by not being able to offer adequate options and adjustments for those presenting major motion deficits or limitations, such as subjects that are wheelchairdependent or that have severely limiting arthrosis.

The correlation coefficient of 0.91 observed between estimated (C-AFQ) and measured (CPET) $\mathrm{VO}_{2}$ max in our 1,000 subjects was quite impressive and higher than the one reported $-\mathrm{r}=0.79-$ in the 212 subjects in the VSAQ's original paper. ${ }^{19}$ Using linear regression analysis, it was possible to show that C-AFQ explained $83 \%$ of the variation in the measured $\mathrm{VO}_{2}$ max. However, it should be pointed out that the standard error of the estimate was 1.54 METs and that, according to our data (Figure 2), the error of estimate was \pm 1 and \pm 2 METs for, respectively, $50 \%$ and $78 \%$ of the subjects. It is also relevant to emphasize that, since the C-AFQ values are based on the subjects' self-report, it was always likely that some of these individual differences could be explained either by self-misevaluation or due to purposeful misinformation - under or overestimation. So, while, in general, aerobic fitness assessed by C-AFQ was a quite good estimator of measured $\mathrm{VO}_{2}$ max, at an individual level, delta METs could be quite high in a relatively large portion of the subjects, with relevant clinical implications, especially when it is known that detraining and training interventions or conditions would rarely be able to produce more than $2 \mathrm{METs}$ changes in measured $\mathrm{VO}_{2} \mathrm{max}$ and, therefore, within this margin of error of estimate.

The application of C-AFQ and its use in planning the individual ramp protocol seemed to have been successful as shown by the profile of CPET duration in which a median value of 11 minutes was observed and the large majority of tests ended after 8 to 15 minutes. An interesting point to comment in our results was the fact that the mean values for sex- and age-predicted and measured (CPET) $\mathrm{VO}_{2}$ max were quite similar, which may reinforce the merit in using Jones' equation ${ }^{29}$ to CLINIMEX's population. However, it is worth noting the huge inter-individual variability that ranged from -9.6 to 8.3 METs or from $28.8 \%$ to $236 \%$ of sex- and agepredicted $\mathrm{VO}_{2}$ max in the 1,000 subjects studied, which clearly indicates the limitation of using sex- and agepredicted $\mathrm{VO}_{2}$ max values for planning individual ramp protocols, much differently than what was observed with the estimated aerobic fitness obtained by C-AFQ. 
The tool most often used in the literature to perform non-exercise estimate of aerobic fitness is the VSAQ. ${ }^{19-21,23}$ Notwithstanding, as briefly mentioned in our introduction, it has several shortcomings or limitations and, as recently confirmed, its association with measured (CPET) $\mathrm{VO}_{2}$ max is only moderate..$^{22}$ For instance, there are several differences between C-AFQ and VASQ that may help to explain why our C-AFQ data presented better association between estimated and measured aerobic fitness than the original VSAQ data.

The first major difference is the range of exercise intensities covered in both questionnaires, with the 0.5 MET interval scale adopted in the lower range of the C-AFQ as compared to 1-MET interval across all VSAQ scales and the extension of scale to $>20$ METs in C-AFQ as compared with the 13-MET maximal limit in the original VSAQ, allowing both severely unfit and fitter subjects to be better quantified and discriminated by the C-AFQ. The second major difference resides in the two-step approach used in C-AFQ versus the single-step approach in VSAQ. Interestingly, although apparently a two-step versus a single-step approach and the list of 63 activities in step 2 versus only 21 activities in the VSAQ would seem to be much more complicated, in practical terms, this was not true. Applying the step 1 of C-AFQ allowed a very simple and straightforward answer. Indeed, the vast majority of the subjects would be answering zones 3 to 6 and, according to sex, age and clinical conditions, an even more limited range could be initially asked by the interviewer. For example, in 50-year-old apparently healthy men, the interviewer could start C-AFQ by asking if they are able to "run, slowly, at least $1 \mathrm{~km}$ or 10 minutes without stopping or walking" (zone 5). If a negative response is given, the interviewed would downgrade to the question in zone 4 - are you able to "run, slowly, one block or 100 meters?," otherwise, in case of a positive response to the first question, the interviewer would upgrade to zone 6 and the question would be if they were able to "run, continuously, for up to 40 minutes" and the questioning would continue until the "best block or zone number" in step 1 is identified and then following to step 2. Having defined the proper block number in the step 2 of C-AFQ, five and twelve activities are listed according to exercise intensities estimated in METs. In this sense, C-AFQ is likely to be easier and faster to apply while still being more precise and more discriminative than VSAQ in identifying the subject's maximal tolerable exercise and in estimating aerobic fitness (please see a demonstration video in supplemental materials).*
Interestingly, the delta METs were quite similar among the four physicians that collaborated with C-AFQ data for this study. This suggests that adequately trained (after mastering the application C-AFQ instructions) health professionals would be able to successfully use C-AFQ in their practice to estimate aerobic fitness.

Finally, several other associations, at varying degrees, were found. For the major study variable - delta METs between estimated (C-AFQ) and measured (CPET) $\mathrm{VO}_{2} \max -$ no clinically relevant influence was found regarding age, sex, height, weight, waist girth, body mass index, major clinical conditions, regular use of ß-blockers, type of CPET's ergometer, SRT scores and maximal muscle power related to body weight. A small association was found between delta METs and relative measured METs expressed as \% sex- and age-predicted METs, indicating a small trend for higher absolute errors in those exercise practitioners at an upper range of aerobic fitness. Indeed, from a clinical perspective, a small error at $>12$ METs is much less relevant than a similar magnitude of error at the lower range of aerobic fitness. Although it was not among the main objectives of the study, the presence of a significant yet moderate association $-\mathrm{r}$ values ranging from 0.63 to $0.71-$ between measured (CPET) aerobic fitness and nonaerobic (SRT or maximal muscle power) fitness, with all these variables clearly and independently associated with all-cause mortality, 2,32,34 is a new finding that should be further explored in upcoming epidemiological studies using CLINIMEX's cohort.

This study has several positive points: 1 ) the prospective design; 2) the large and varied sample in terms of sex, clinical condition, ergometer and levels of aerobic fitness (as often seen in clinical practice); 3) all data collected under well-controlled conditions by only four specialized physicians; 4) the use of a gold standard for criterion validity and; 5$)$ the possibility of assessing the influence of several other variables, including results of two assessment tools of non-aerobic physical fitness. On the other hand, the study also has some limitations: 1) the sample was primarily comprised of white subjects with high educational level and/or upper socioeconomical class; and 2) only four specialized physicians applied the C-AFQ. Both limitations could have influenced the external validity of our results and only future studies with other populations and a larger number of applicants will be able to show if the present results can be generalized or not. 


\section{Conclusions}

In summary, we concluded that: a) C-AFQ was successfully applied in a large sample of subjects who voluntarily underwent $\mathrm{CPET}$; $2 \mathrm{~b}$ ) $\mathrm{C}-\mathrm{AFQ}$ was valid to estimate aerobic fitness by four specialized physicians and could be used as a non-exercise alternative when maximal CPET is unavailable or cannot be performed; c) C-AFQ can be very useful to support the planning of individualized CPET ramp protocols; d) C-AFQ's error of estimate of $\mathrm{VO}_{2}$ max was clinically too high in some of the subjects, with a small trend of larger errors in the upper extremes of aerobic fitness; e) C-AFQ's error of the estimate of $\mathrm{VO}_{2}$ max was largely independent of age, sex, major anthropometric measurements, clinical condition, regular use of $B$-blockers, type of CPET ergometer and non-aerobic physical fitness levels.

\section{Author contributions}

Conception and design of the research: Araujo CG, Castro CL, Franca JF, de Souza e Silva CG. Acquisition of data: Araujo CG, Castro CL, Franca JF, de Souza e Silva CG. Analysis and interpretation of the data: Araujo CG, Castro CL, Franca JF, de Souza e Silva CG. Statistical analysis: Araujo CG, de Souza e Silva CG. Obtaining financing: Araujo CG. Writing of the manuscript: Araujo CG. Critical revision of the manuscript for intellectual content: Araujo CG, Castro CL, Franca JF, de Souza e Silva CG.

\section{Potential Conflict of Interest}

No potential conflict of interest relevant to this article was reported.

\section{Sources of Funding}

This study was partially funded by CNPq/FAPERJ.

\section{Study Association}

This study is not associated with any thesis or dissertation work.

\section{Ethics approval and consent to participate}

This study was approved by the Ethics Committee of the SUPREMA under the protocol number 218/2011. All the procedures in this study were in accordance with the 1975 Helsinki Declaration, updated in 2013. Informed consent was obtained from all participants included in the study.

\section{References}

1. Khan H, Jaffar N, Rauramaa R, Kurl S, Savonen K, Laukkanen JA. Cardiorespiratory fitness and nonfatalcardiovascular events: A population-based follow-up study. Am Heart J. 2017 Feb;184:55-61.

2. Kodama S, Saito K, Tanaka S, Maki M, Yachi Y, Asumi M, et al. Cardiorespiratory fitness as a quantitative predictor of all-cause mortality and cardiovascular events in healthy men and women: a meta-analysis. JAMA. 2009;301(19):2024-35.

3. Mandsager K, Harb S, Cremer P, Phelan D, Nissen SE, Jaber W Association of Cardiorespiratory Fitness With Long-term Mortality Among Adults Undergoing Exercise Treadmill Testing. JAMA Netw Open. 2018;1(6):e183605.

4. Harber MP, Kaminsky LA, Arena R, Blair SN, Franklin BA, Myers J, et al. Impact of Cardiorespiratory Fitness on All-Cause and Disease-Specific Mortality: Advances Since 2009. Prog Cardiovasc Dis. 2017;60(1):11-20.

5. Kaminsky LA, Arena R, Ellingsen O, Harber MP, Myers J, Ozemek C, et al. Cardiorespiratory fitness and cardiovascular disease - The past, present, and future. Prog Cardiovasc Dis. 2019;62(2):86-93.

6. Lakka TA, Laukkanen JA, Rauramaa R, Salonen R, Lakka HM, Kaplan GA, et al. Cardiorespiratory fitness and the progression of carotid atherosclerosis in middle-aged men. Ann Intern Med. 2001;134(1):12-20.

7. Ross R, Blair SN, Arena R, Church TS, Despres JP, Franklin BA, et al. Importance of Assessing Cardiorespiratory Fitness in Clinical Practice:

A Case for Fitness as a Clinical Vital Sign: A Scientific Statement From the American Heart Association. Circulation. 2016;134(24):e653-e99.

8. Araújo CGS, Herdy AH, Stein R. Medida do consumo máximo de oxigênio: valioso marcador biológico na saúde e na doença. Arq Bras Cardiol. 2013;100(4):e51-e63.

9. Laukkanen JA, Rauramaa R, Makikallio TH, Toriola AT, Kurl S. Intensity of leisure-time physical activity and cancer mortality in men. Br J Sports Med. 2011;45(2):125-9.

10. Myers J, Prakash M, Froelicher V, Do D, Partington S, Atwood JE. Exercise capacity and mortality among men referred for exercise testing. N Engl J Med. 2002;346(11):793-801.

11. Ehrman JK, Brawner CA, Al-Mallah MH, Qureshi WT, Blaha MJ, Keteyian SJ. Cardiorespiratory Fitness Change and Mortality Risk Among Black and White Patients: Henry Ford Exercise Testing (FIT) Project. Am J Med. 2017;130(10):1177-83.

12. Laukkanen JA, Zaccardi F, Khan H, Kurl S, Jae SY, Rauramaa R. Longterm Change in Cardiorespiratory Fitness and All-Cause Mortality: A Population-Based Follow-up Study. Mayo Clin Proc. 2016;91(9):1183-8.

13. Imboden MT, Harber MP, Whaley MH, Finch WH, Bishop DL, Fleenor BS, et al. The Association between the Change in Directly Measured Cardiorespiratory Fitness across Time and Mortality Risk. Prog Cardiovasc Dis. 2019;62(2):157-62. 
14. Herdy AH, Ritt LE, Stein R, Araujo CG, Milani M, Meneghelo RS, et al. Teste cardiopulmonar de exercício: fundamentos, aplicabilidade e interpretação. Arq Bras Cadiol. 2016;107(5):467-81.

15. Kaminsky LA, Myers J, Arena R. Determining Cardiorespiratory Fitness With Precision: Compendium of Findings From the FRIEND Registry. Prog Cardiovasc Dis. 2019;62(1):76-82.

16. Guazzi M, Adams V, Conraads V, Halle M, Mezzani A, Vanhees L, et al. EACPR/AHA Joint Scientific Statement. Clinical recommendations for cardiopulmonary exercise testing data assessment in specific patient populations. Eur Heart J. 2012;33(23):2917-27.

17. Sadik J, Myers J, Froelicher V. A modified nomogram for ramp treadmill testing using the Veterans Specific Activity Questionnaire. Am J Cardiol. 2014;114(5):803-5.

18. Araújo DSMS, Araújo CGS. Autopercepção das variáveis da aptidão física. Rev Bras Med Esporte. 2002;8(2):37-49.

19. Myers J, Do D, Herbert W, Ribisl P, Froelicher VF. A nomogram to predict exercise capacity from a specific activity questionnaire and clinical data. Am J Cardiol. 1994;73(8):591-6.

20. Gawecki F, Myers J, Shovlin CL. Veterans Specific Activity Questionnaire (VSAQ): a new and efficient method of assessing exercise capacity in patients with pulmonary arteriovenous malformations. BMJ Open Respir Res. 2019;6(1):e000351.

21. Myers J, Bader D, Madhavan R, Froelicher V. Validation of a specific activity questionnaire to estimate exercise tolerance in patients referred for exercise testing. Am Heart J. 2001;142(6):1041-6.

22. Teren A, Zachariae S, Beutner F, Ubrich R, Sandri M, Engel C, et al. Incremental value of Veterans Specific Activity Questionnaire and the YMCA-step test for the assessment of cardiorespiratory fitness in population-based studies. Eur J Prev Cardiol. 2016;23(11):1221-7.

23. Maranhao-Neto G de A, Leon AC, Farinatti Pde T. Validity and equivalence of the Portuguese version of the Veterans Specific Activity Questionnaire. Arq Bras Cardiol. 2011;97(2):130-5.

24. Domingues Gde B, Gallani MC, Gobatto CA, Miura CT, Rodrigues RC, Myers J. Cultural adaptation of an instrument to assess physical fitness in cardiac patients. Rev Saude Publica. 2011;45(2):276-85.
25. Araujo CG, Scharhag J. Athlete: a working definition for medical and health sciences research. Scand J Med Sci Sports. 2016;26(1):4-7.

26. Ricardo DR, De Almeida MB, Franklin BA, Araujo CG. Initial and final exercise heart rate transients: Influence of gender, aerobic fitness, and clinical status. Chest. 2005;127(1):318-27.

27. Ramos PS, Araujo CG. Cardiorespiratory optimal point during exercise testing as a predictor of all-cause mortality. Rev Port Cardiol. 2017;36(4):261-9.

28. de Souza e Silva CG, Araújo CG. Equações específicas por sexo para estimativa do consumo máximo de oxigênio em cicloergometria. Arq Bras Cardiol. 2015;105(4):381-9.

29. Jones NL, Campbell EK, Edwards RH, Robertson DG. Clinical exercise testing. Philadelphia: WB Saunders; 1975.

30. Ainsworth BE, Haskell WL, Leon AS, Jacobs DR Jr, Montoye HJ, Sallis JF, et al. Compendium of physical activities: classification of energy costs of human physical activities. Med Sci Sports Exerc. 1993;25(1):71-80.

31. De Souza e Silva CG, Franklin BA, Araújo CG. Influence of central obesity in estimating maximal oxygen uptake. Clinics. 2016;71(11):629-34.

32. Brito LB, Ricardo DR, Araújo DS, Ramos PS, Myers J, Araujo CG. Ability to sit and rise from the floor as a predictor of all-cause mortality. Eur J Prev Cardiol. 2014;21(7):892-8.

33. Araujo CGS, Castro CLB, Franca JFC, Araujo DS. Sitting-rising test: Sexand age-reference scores derived from 6141 adults. Eur J Prev Cardiol. 2019: 2047487319847004.[Epub ahead of print]

34. Araujo CG, Castro CL, Franca JF, Laukkanen JA, Hamar D, Myers J. Muscle power in upright row movement: predictor of all-cause mortality in individuals between 41 and 85 years of age: preliminary results. Eur J Prev Cardiol. 2019;26(Suppl 6):P459.

\section{*Supplemental Materials}

For additional information, please click here. 